Check for updates

Cite this: Mater. Adv., 2022, 3, 2417

Received 16th November 2021, Accepted 4th January 2022

DOI: 10.1039/d1ma01079d

rsc.li/materials-advances

\title{
Coating liposomes with ring-like PEG: the synthesis and stealth effect of cholesterol-PEG-cholesterol
}

\author{
Yufang Wang, (D) ${ }^{a}$ Jianzhu Wang, ${ }^{a}$ Mingshuang Sun, ${ }^{a}$ Jiyan Zhang $^{\mathrm{b}}$ and \\ Yanping $\mathrm{Bi}$ (D) *a
}

\begin{abstract}
Escaping from the immune system to achieve a long circulation time is an essential prerequisite for targeting liposomes. Monomethoxy-poly(ethylene glycol)-distearoylphosphatidylethanolamine (mPEGDSPE) is the most commonly used stealth polymer for long circulating liposomes. With its monomethoxy end moving freely on the liposomal surface, the linear PEG chains of $\mathrm{mPEG-DSPE}$ construct a hydration layer to repel complement proteins and thus evade immune clearance. We describe in this work a new kind of PEG derivative whose two ends were grafted with cholesterol (cholesterol-PEG-cholesterol, (PC). According to the in vitro and in vivo results, the presence of CPC in liposomes was shown to reduce the uptake by phagocyte cells while extending the circulation time. The stealth performance of CPC liposomes is closely related to the molecular weight of PEG moieties. CPC $6 \mathrm{k}_{\mathrm{k}}$ performed better than $\mathrm{CPC}_{2 \mathrm{k}}, \mathrm{CPC}_{4 \mathrm{k}}$ and the commonly used $\mathrm{mPEG}_{2 \mathrm{k}}-\mathrm{DSPE}$. CPC on the liposomal surface would not let the free end of PEG act as the immune recognition site, meanwhile, the PEG chain of CPC tends to adopt a twisted ring-like conformation rather than a linear conformation, which is considered to be associated with the stealth effect. This finding adds to the range of stealth liposomes and may be valuable for developing new drug carriers.
\end{abstract}

\section{Introduction}

Nanoscale drug carriers injected intravenously unavoidably suffer from the rapid uptake by the mononuclear phagocyte system (MPS) before reaching the target site, which causes the off-target effect of drug delivery. The size and surface properties of nanocarriers have been regarded as the most important factors that influence MPS uptake. ${ }^{1}$ Opsonization induced by the adsorption of circulating proteins onto the surface of nanocarriers plays a crucial role in MPS recognition. ${ }^{2}$ Therefore a long circulation time can be achieved by decorating nanocarriers with hydrophilic polymers such as poly(ethylene glycol) (PEG), poly(2-oxazoline) (POx) ${ }^{3}$ and hyaluronic acid, ${ }^{4}$ among which PEG is the most widely-used polymer. ${ }^{5-7}$

PEG chains repel proteins due to their high hydrophilicity and flexibility, so an intact PEG hydration layer with enough thickness on the nanoparticle surface is essential for preventing the adsorption of plasma proteins. The length (molecular weight, MW), density and conformation of PEG chains, which correlate with each other on the nanoparticle surface, strongly

\footnotetext{
${ }^{a}$ School of Pharmaceutical Sciences, Shandong First Medical University, China. E-mail: biyanpingtsmc@163.com

${ }^{b}$ Tai An TSCM Hospital, China
}

affect the circulation time of nanoparticles. A reasonably higher MW of PEG chains leads to a thicker hydration layer on the nanoparticle surface and thus a lower level of opsonization. ${ }^{2,8,9}$ A low ratio of surface coverage may bring about the interaction between the proteins and exposed surface.

The PEG chains on the particle surface could show a "mushroom" or "brush" conformation depending on their grafting density. In order to achieve a better stealth-like behavior, the PEG conformation needs to be optimized between the "mushroom" and "brush" because the over-high coverage ratio and density, which result in a "brush" conformation, may reduce the flexibility of PEG chains. ${ }^{1,2}$ In addition, there is easily overlooked relevance between the end-group of PEG chains and MPS uptake. Compared with hydroxyl- or mannoside-terminated PEG, methoxy-terminated PEG can protect nanocarriers from MPS recognition more effectively, ${ }^{10}$ which implies that the end group of PEG is an important recognition site for MPS clearance.

PEGylated liposomes have been widely used as a kind of long circulating nanocarrier for drug targeting especially after the approval of Doxil ${ }^{\circledR}$ by the FDA. It is necessary for a PEG chain to anchor at least one of its two ends onto the surface of liposomes, so that a stable hydration layer can be preserved. In previous reports, the other end of the PEG, usually capped 

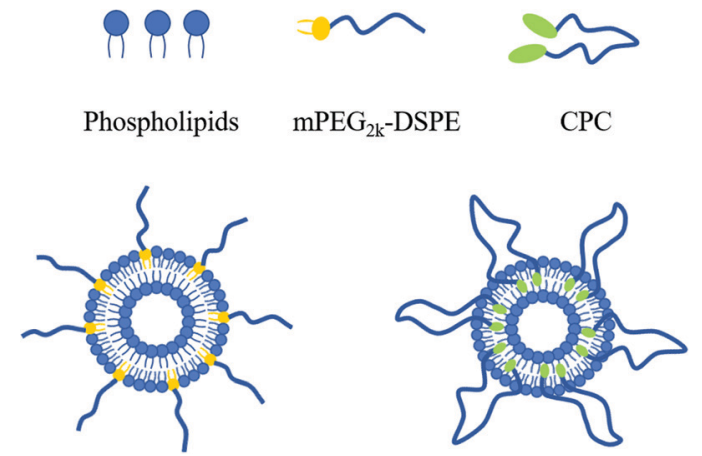

Fig. 1 Schematic representation of linear (left) and ring-like (right) conformations of PEG moieties on the liposomal surface.

with a methoxy group or a targeting ligand, can move freely within the hydration layer. For example, monomethoxypoly(ethylene glycol)-distearoylphosphatidylethanolamine (mPEGDSPE), the most commonly used stealth polymer for stealth liposomes, ${ }^{11}$ could be fastened to a liposome by inserting its distearoyl group into a phospholipid bilayer. As compared to its homologues, $\mathrm{PEG}_{2 \mathrm{k}}-\mathrm{DSPE}$ attracts more attention due to its low toxicity and stabilization effect on liposomes. ${ }^{12-14}$ The superiorities of the $\mathrm{PEG}_{2 \mathrm{k}}-\mathrm{DSPE}$ unit in fabricating stealth liposomes are rooted in its suitable hydrophile-lipophile balance (HLB) value which is decided by the MW ratio of PEG to DSPE. That is to say, extending the PEG chain might not bring about a better stealth effect but cause unpredictable toxicity or self-assembly of mPEG-DSPE.

If the other end of MPEG-DSPE was also grafted with a lipophilic group, the MW of the PEG chain could be doubled with the HLB value remaining unchanged. From a thermodynamics perspective, both ends of the lipophilic groups tend to insert into a liposomal membrane to construct a ring-like PEG chain (Fig. 1). The unique ring-like conformation of the PEG chain would endow liposomes with improved properties. Cholesterol can be a suitable alternative to anchoring into liposomal membranes, ${ }^{15}$ so in this work, cholesterol molecules were attached to both ends of PEG to obtain cholesterol-PEGcholesterol (CPC). Subsequently, CPC-containing liposomes were prepared and their physicochemical properties, cellular uptake and pharmacokinetic behaviors were also studied.

\section{Materials and methods}

\subsection{Materials}

Cholesterol was supplied by Source Leaf Biology Co., Ltd. $N, N^{\prime}$ Dicyclohexylcarbodiimide (DCC) and 4-dimethylaminopyridine (DMAP) were purchased from Shanghai Aladdin Biochemical Technology Co., Ltd. mPEG $_{2 \mathrm{k}}-\mathrm{DSPE}$ and lecithin were purchased from AVT Pharmaceutical Tech Co., Ltd (Shanghai, China). Methyl thiazolyl tetrazolium (MTT) was purchased from Hebei Bailingwei Superfine Material Co., Ltd (Beijing, China). Coumarin-6 (Cou-6) was obtained from Sigma-Aldrich (Shanghai) Trading Co., Ltd. Dulbecco's modified eagle medium (DMEM) was purchased from Jiangsu KGI Biotechnology Co., Ltd (China). PEG of different MWs (2 kD, $4 \mathrm{kD}$ and $6 \mathrm{kD})$, succinic anhydride
(SA), and other chemical regents were purchased from Sinopharm Chemical Reagent Co., Ltd (Shanghai, China).

\subsection{Synthesis and characterization of CPC}

$\mathrm{PEG}_{2 \mathrm{k}}, \mathrm{PEG}_{4 \mathrm{k}}$ and $\mathrm{PEG}_{6 \mathrm{k}}$ were used to synthesize $\mathrm{CPC}$ of different MWs by the same two-step reaction. PEG of $5 \mathrm{mmol}$ was dissolved into $20 \mathrm{~mL}$ of toluene and heated to $85{ }^{\circ} \mathrm{C}$ to distill the toluene-water azeotrope. Then $50 \mathrm{mmol}$ SA was added and the solution was stirred for $3 \mathrm{~h}$ at $110{ }^{\circ} \mathrm{C}$. After the addition of $20 \mathrm{~mL}$ of water (to remove the residual of SA), the reaction system was extracted three times with dichloromethane. The dichloromethane layer was washed in triplicate using water, dehydrated using anhydrous sodium sulfate for $24 \mathrm{~h}$, and poured into $200 \mathrm{~mL}$ of petroleum ether to obtain PEG diacid as a precipitate. The obtained PEG diacid precipitate was analyzed by thin-layer chromatography (TLC) eluted with dichloromethane-methanol-aqueous ammonia $(8.5: 1.5: 0.1, \mathrm{v} / \mathrm{v} / \mathrm{v})$.

In the second step, PEG diacids $(2 \mathrm{mmol})$, cholesterol $(8 \mathrm{mmol})$, DMAP (1.955 g) and DCC (3.3 g) were dissolved into $60 \mathrm{~mL}$ of anhydrous dichloromethane. The mixture was stirred under nitrogen at room temperature for $24 \mathrm{~h}$ and then filtered. The filtrate was washed with $0.1 \mathrm{M} \mathrm{HCl}, 1 \%$ aqueous ammonia and distilled water in turn until $\mathrm{pH}=7$, then was dehydrated using anhydrous sodium sulfate for $24 \mathrm{~h}$ and re-precipitated using petroleum ether to obtain CPC. The detailed synthetic scheme is illustrated in Fig. 2.

Fourier transform infrared (FT-IR) spectra of CPC samples were recorded using a Shimadzu IRAffinity-1 spectrometer at frequencies ranging from 400 to $4000 \mathrm{~cm}^{-1}$ with a $4 \mathrm{~cm}^{-1}$ resolution. Typically, $1 \mathrm{mg}$ of each sample was mixed with $20 \mathrm{mg} \mathrm{KBr}$ and compressed into tablets before determination. ${ }^{1} \mathrm{H}$ and ${ }^{13} \mathrm{C}$ nuclear magnetic resonance (NMR) spectra of samples in $\mathrm{DMSO}-d_{6}$ were recorded on a Bruker Avance NEO400 at frequencies of $400 \mathrm{MHz}$ at $298 \mathrm{~K}$.

\subsection{Preparation and characterization of CPC liposomes}

CPC synthesized with $\mathrm{PEG}_{2 \mathrm{k}}, \mathrm{PEG}_{4 \mathrm{k}}$ and $\mathrm{PEG}_{6 \mathrm{k}}\left(\mathrm{CPC}_{2 \mathrm{k}}, \mathrm{CPC}_{4 \mathrm{k}}\right.$ and $\mathrm{CPC}_{6 \mathrm{k}}$ ) were respectively used as stealth polymers to prepare liposomes along with lecithin. Typically, $100 \mathrm{mg}$ of lecithin and $10 \mathrm{mg}$ of CPC were dissolved in a mixed solvent consisting of ethyl acetate and ethanol $(1: 1)$ before $1000 \mu \mathrm{L}$ of Cou-6 solution $\left(100 \mu \mathrm{g} \mathrm{mL}{ }^{-1}\right)$ in dichloromethane was added. The organic solvent was removed in a rotary evaporator to yield a thin lipid film. Then the film was hydrated with $10 \mathrm{~mL}$ of physiological saline and treated with a high pressure homogenizer at 10 000-15000 psi for $5 \mathrm{~min}$.

The size, polydispersity index (PDI) and zeta potential of liposomes were analyzed with a Malvern Zetasizer Nano ZSE. For each test, $100 \mu \mathrm{L}$ of liposomal suspension was mixed with $1.000 \mathrm{~mL}$ of ultrapure water at $25{ }^{\circ} \mathrm{C}$. A JEM-1200 EX II transmission electron microscope (TEM, JEOL Ltd.) was used to observe the morphology of liposomes. Before observation, about $5 \mu \mathrm{L}$ of diluted liposomal suspension was dripped onto a carbon-film-coated copper grid and dried in a vacuum, and then stained with $2.0 \%(\mathrm{w} / \mathrm{w})$ phosphotungstic acid solution for $2 \mathrm{~min}$. 
<smiles>CC(C)OCCC(C)(C)OCC(C)(C)OCCC(=O)OC(C)(C)CCC(=O)O</smiles>

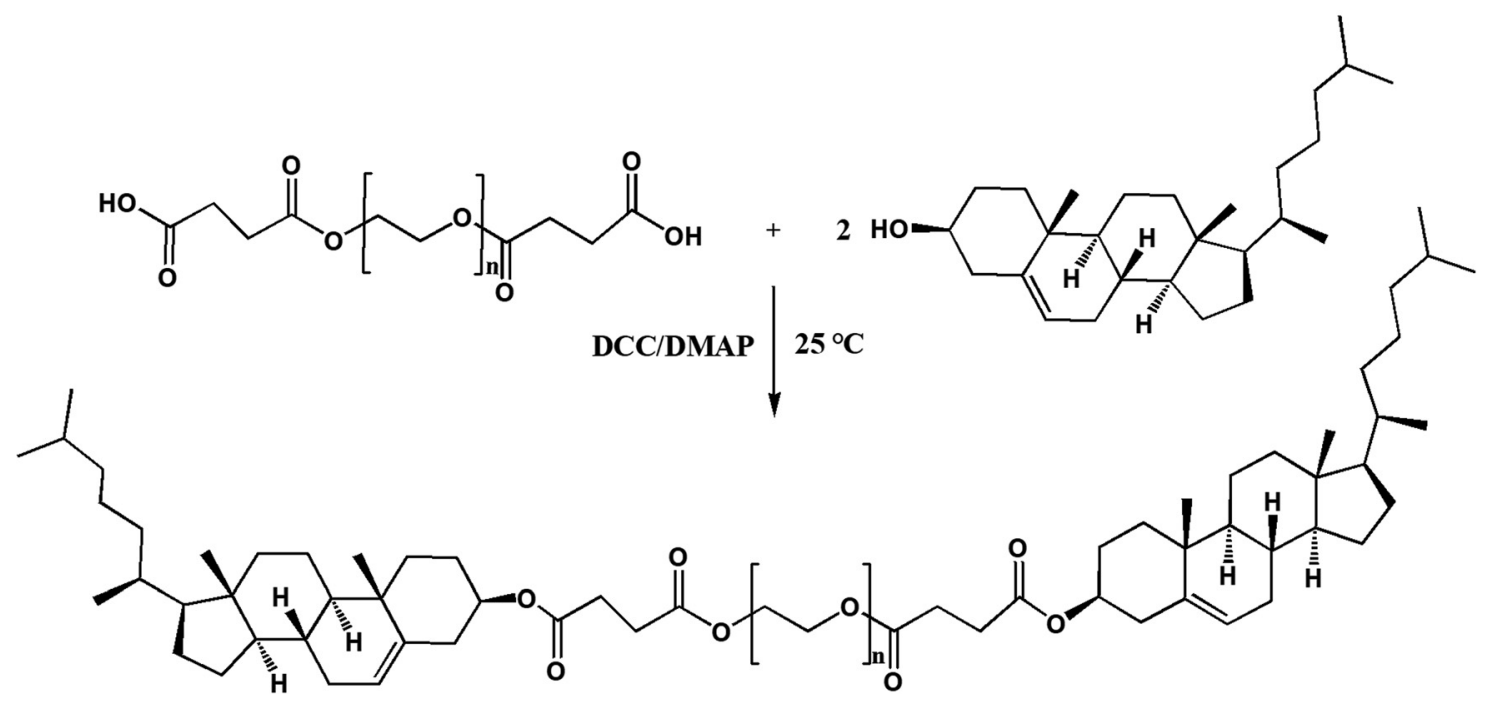

Fig. 2 Synthesis procedure of CPC.

\subsection{Determination of Cou-6}

The concentration of Cou- 6 in liposomes or plasma was determined by high performance liquid chromatography (HPLC). The HPLC system was composed of a Shimadsu LC-20AD pump and a RF-20A fluorescent detector. A GL-sciences Intersil column (ODS-3, $5 \mu \mathrm{m}$, $4.6 \times 200 \mathrm{~mm}$ ) was used with the mobile phase of acetonitrile/water $(80 / 20)$ at a flow rate of $1.000 \mathrm{~mL} \mathrm{~min}^{-1}$. The excitation and emission wavelengths of detection are $466 \mathrm{~nm}$ and $504 \mathrm{~nm}$, respectively. Each sample of liposomes or plasma of $100 \mu \mathrm{L}$ was mixed intensively with $400 \mu \mathrm{L}$ of acetonitrile and then centrifuged at 10000 rpm for $10 \mathrm{~min}$ before loading into the HPLC system.

The concentration of Cou- 6 was calculated using a standard curve. In the range of $0.08-16 \mathrm{ng} \mathrm{mL}^{-1}$, the concentration of Cou-6 $\left(Y, \mathrm{ng} \mathrm{mL}^{-1}\right)$ correlated well with its peak area $(X)$ : $Y=1.3469 X+0.0358\left(R^{2}=0.9999\right)$. Three samples of Cou-6 at concentrations of $0.4,4.0$ and $8.0 \mathrm{ng} \mathrm{mL}{ }^{-1}$ were selected for methodology validation. The recovery of this method ranges from $97 \%$ to $105 \%$ and the relative standard deviation (RSD) is smaller than $2 \%$ for $48 \mathrm{~h}$.

\subsection{Drug loading and leakage rate of Cou-6 in liposomes}

The drug loading of Cou- 6 in liposomes was calculated as follows:

$$
\text { Drug loading }=\frac{\text { Cou- } 6 \text { amount in liposome }}{\text { added amount of Cou- } 6} \times 100 \%
$$

Before acetonitrile pretreatment and HPLC analysis (see 2.4), the samples of liposomes were centrifuged at $3000 \mathrm{rpm}$ for $5 \mathrm{~min}$ and diluted with ultrapure water 1000 times.

In order to investigate the leakage of Cou- 6 in cell culture media, the dialysis test was conducted at $37{ }^{\circ} \mathrm{C}$ in a horizontal diffusion cell. The donor and receptor compartments were separated by a dialysis membrane $(3.5 \mathrm{kD})$ and filled with $2.0 \mathrm{~mL}$ of liposomes and $7.0 \mathrm{~mL}$ of culture media, respectively. After magnetic stirring (100 rpm) for $24 \mathrm{~h}, 100 \mu \mathrm{L}$ of the culture media was withdrawn and mixed with $400 \mu \mathrm{L}$ of acetonitrile for HPLC determination. The leakage rate was calculated by the following equation:

Leakage rate

Cou-6 amount in culture media

$=\overline{\text { Cou- } 6 \text { amount in liposome }+ \text { Cou- } 6 \text { amount in culture media }}$

$\times 100 \%$

\subsection{In vitro cytotoxicity}

Murine macrophage cell line Raw 264.7 was cultured in a highglucose DMEM substrate containing $10 \%$ fetal bovine serum in an incubator under a humidified atmosphere of $5.0 \% \mathrm{CO}_{2}$ at $37{ }^{\circ} \mathrm{C}$. The in vitro cytotoxicity of different kinds of liposomes was assessed by MTT assay. Briefly, Raw 264.7 cells were seeded in a 96-well microplate at a density of $10^{4}$ cells per well and incubated overnight to allow them to attach. Liposomal suspensions were added to arrive at concentrations ranging from 0.1 to $10 \mu \mathrm{g} \mathrm{mL} \mathrm{m}^{-1}$ (here the concentration refers to CPC, $\mathrm{mPEG}_{2 \mathrm{k}}-\mathrm{DSPE}$ or cholesterol for each kind of liposome) and incubated for a further $48 \mathrm{~h}$. Next, $20 \mu \mathrm{L}$ of MTT solution $\left(5 \mathrm{mg} \mathrm{mL}{ }^{-1}\right)$ was added into each well and incubated with cells for $4 \mathrm{~h}$. Finally, the medium was replaced with $150 \mu \mathrm{L}$ of dimethyl sulfoxide and incubated for $10 \mathrm{~min}$. The relative cell viability for each well was calculated by using the absorbance at $490 \mathrm{~nm}$.

\subsection{Cellular uptake studies}

The cellular uptake amount of liposomes was analyzed by flow cytometry. First, Raw 264.7 cells were cultured in 24 -well plates at a density of $2 \times 10^{5}$ cells per well until approximately $70 \%$ 
confluence. Taking DMSO solution of Cou-6 as the control, Cou-6 labeled liposomes were diluted with serum-free medium to achieve a Cou- 6 concentration of $0.03 \mu \mathrm{g} \mathrm{mL}{ }^{-1}$. Then the serum-free medium containing Cou- 6 labeled liposomes or free Cou- 6 liposomes were used to replace the original medium and incubated for 1, 4, 12 and $24 \mathrm{~h}$, respectively. After that, the cells were washed three times with phosphate-buffered saline (PBS) solution, digested with trypsin, collected and resuspended in $1.0 \mathrm{~mL}$ of medium. Finally, the fluorescence intensity of the cells was measured using a BD FASCVerse ${ }^{\mathrm{TM}}$ flow cytometer.

\subsection{Pharmacokinetic (PK) studies}

Twenty healthy male SD rats, $270-300 \mathrm{~g}$, were randomly divided into 4 groups and fasted for 12 hours with drinking water before the experiment. Liposomes with Cou-6 as fluorescent probes were injected through the tail vein at a Cou- 6 dosage of $3.7 \mu \mathrm{g}$ per $100 \mathrm{~g}$. Blood samples of $0.5 \mathrm{~mL}$ were collected into anticoagulant tubes from tail veins at 5, 15, 30, 60, 120, 240 and $360 \mathrm{~min}$ and centrifuged at $3000 \mathrm{rpm}$ for $10 \mathrm{~min}$ to obtain plasma. The concentration of Cou- 6 in the plasma was determined by HPLC (2.4) and the pharmacokinetic parameters were calculated using DAS 2.0 software. All experiments were performed in compliance with the relevant laws and guidelines of Shandong First Medical University and approved by the institutional committee.

\subsection{Statistical analysis}

Statistical analysis was conducted using GraphPad Prism (9.0.0). The data were presented as the mean \pm standard deviation (SD) from at least three independent measurements. Statistical significances were assessed using the Student $t$-test. A probability value $(p)$ of less than 0.05 was considered statistically significant.

\section{Results and discussion}

\subsection{Structure confirmation of CPC}

With the assistance of bridging agent SA, cholesterol moieties were grafted onto both ends of the PEG chain to produce CPC. PEG diacid and equimolar amounts of cholesterol will produce mono-cholesteryl PEG (Chol-PEG), which can be easily distinguished from CPC with silica TLC. Actually, excess SA and cholesterol were used here to make sure that CPC rather than Chol-PEG is produced. The structures of CPCs with different MWs have been confirmed further using FT-IR, ${ }^{1} \mathrm{H}$ and ${ }^{13} \mathrm{C}$ NMR spectra. The symmetric deformation vibration of carbonyl in SA moieties at $1734 \mathrm{~cm}^{-1}$ and the $\mathrm{C}=\mathrm{C}$ stretching vibrations of the cholesterol moiety at $1655 \mathrm{~cm}^{-1}$ appeared in the FT-IR spectra of $\mathrm{CPC}_{2 \mathrm{k}}, \mathrm{CPC}_{4 \mathrm{k}}$ and $\mathrm{CPC}_{6 \mathrm{k}}$ (Fig. 3). In the ${ }^{1} \mathrm{H}-\mathrm{NMR}$ spectra (Fig. 4) of CPCs, the characteristic peaks of the cholesterol moiety appeared at 5.34 ppm $(\mathrm{C}=\mathrm{C})$ and 0.5-2.1 ppm $\left(\mathrm{CH}_{3}-\right.$ and $-\mathrm{CH}_{2}-$ ), while the methylene groups of the SA moiety were found at $2.54 \mathrm{ppm}$. In the ${ }^{13} \mathrm{C}-\mathrm{NMR}$ spectra (Fig. 5), signals of the two carbonyl groups of succinic acid moieties were observed at

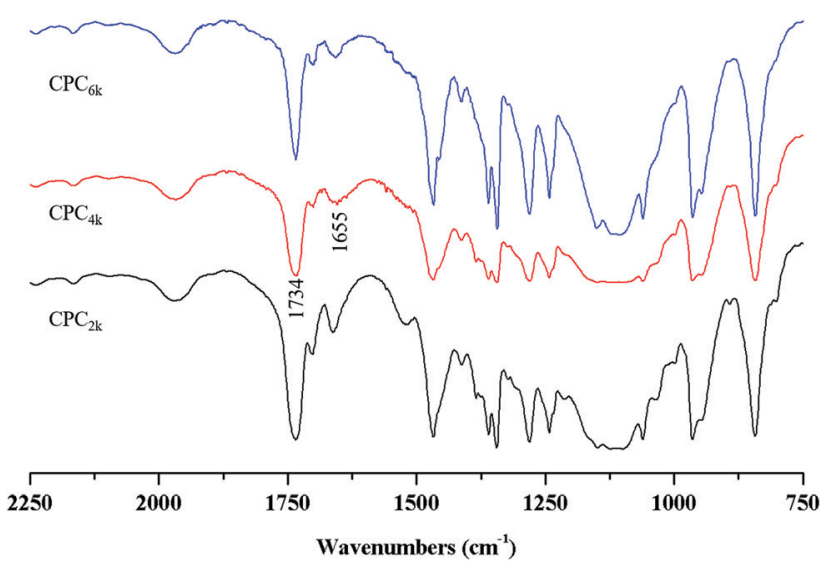

Fig. 3 FTIR spectra of $\mathrm{CPC}_{2 \mathrm{k}}, \mathrm{CPC}_{4 \mathrm{k}}$ and $\mathrm{CPC}_{6 \mathrm{k}}$.

172.49 and $171.67 \mathrm{ppm}$ while signals of the unsaturated carbons of cholesterol moieties were observed at 139.95 and 122.35 ppm.

\subsection{Characterization of liposomes}

The average diameters of most of the CPC liposomes were within the range of 110-140 $\mathrm{nm}$ (Table 1). Compared with the naked liposome (F1) and $\mathrm{mPEG}_{2 \mathrm{k}}-\mathrm{DSPE}$ liposome (F2), there was a significant increase in size for most of the CPC liposomes. Theoretically, PEG chains could construct a hydration layer on the liposome surface, and the thickness of the hydration layer depends on the MW of PEG chains. In other words, a higher MW of PEG would bring about a thicker hydration layer, hence a larger size of liposomes. However, the size decreased with the increase of MW of PEG moieties when a given weight of CPC was used (F7-F9). It is reasonable to assume that the liposomal size was dominated by the HLB value of CPC rather than the thickness of the hydration layer. However, with a given molar amount of CPC used, the liposome size increased with the increase of MW of PEG moieties (F3-F5), that is to say, the size increased with the weight of CPC used. In short, the addition of CPC might increase or decrease the size of liposomes depending on the relative amount of CPC, MW of PEG moieties, hydration volume and homogenization conditions.

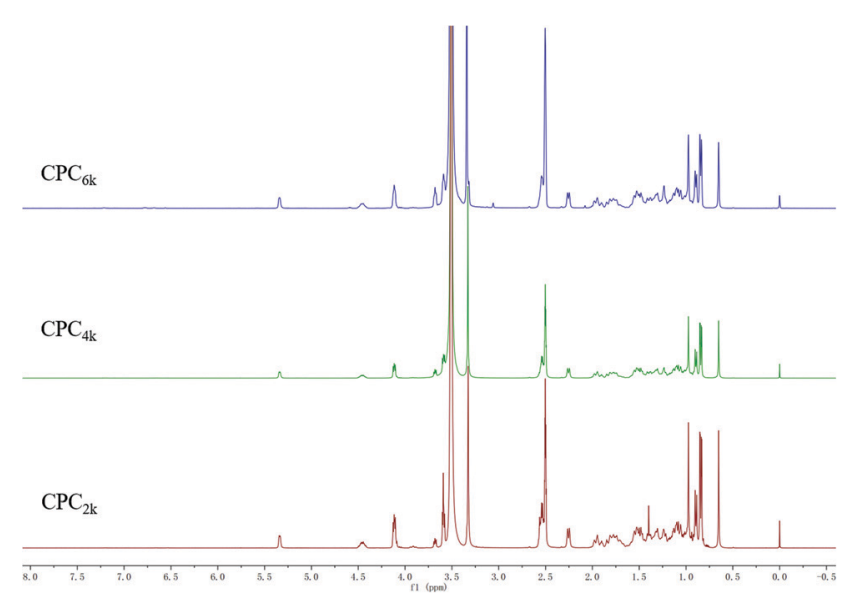

Fig. $4{ }^{1} \mathrm{H}-\mathrm{NMR}$ spectra of $\mathrm{CPC}_{2 \mathrm{k}}, \mathrm{CPC}_{4 \mathrm{k}}$ and $\mathrm{CPC}_{6 \mathrm{k}}$. 


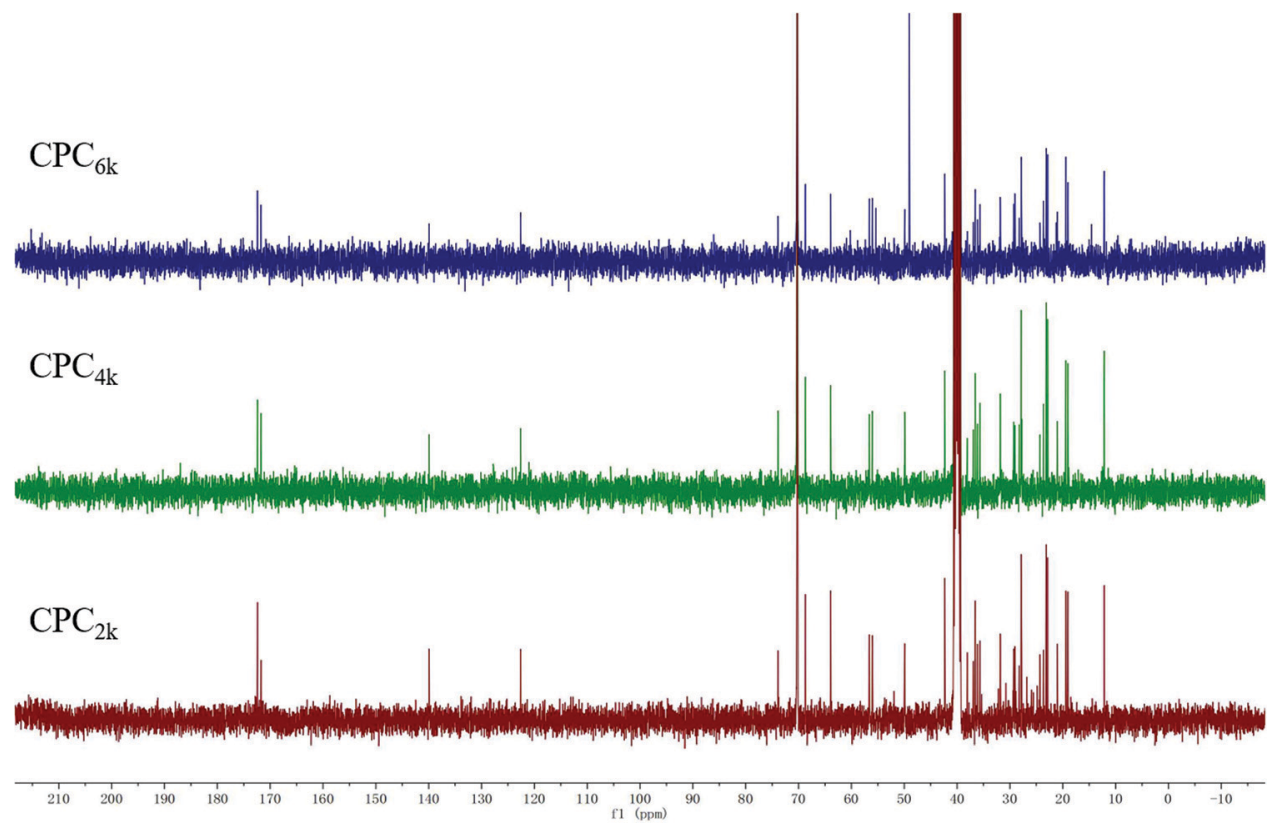

Fig. $5{ }^{13} \mathrm{C}-\mathrm{NMR}$ spectra of $\mathrm{CPC}_{2 \mathrm{k}}, \mathrm{CPC}_{4 \mathrm{k}}$ and $\mathrm{CPC}_{6 \mathrm{k}}$.

Table 1 Particle size, PDI, zeta potential and drug loading of various liposomes

\begin{tabular}{llllll}
\hline Number & Formulation & Hydrodynamic size $(\mathrm{nm})$ & PDI & Zeta potential $(\mathrm{mV})$ & Drug loading $(\%, \mathrm{w} / \mathrm{w})$ \\
\hline F1 & Cholesterol, $2.5 \mathrm{mg}$ & $97.17 \pm 1.39$ & $0.180 \pm 0.011$ & $-18.17 \pm 1.92$ & 85.93 \\
F2 & mPEG $_{2 \mathrm{k}}-\mathrm{DSPE}, 4.00 \mathrm{mg}(1.426 \mu \mathrm{mol})$ & $92.44 \pm 2.35$ & $0.244 \pm 0.008$ & $-13.06 \pm 1.41$ & 86.78 \\
F3 & $\mathrm{CPC}_{2 \mathrm{k}}, 4.24 \mathrm{mg}$ of $(1.426 \mu \mathrm{mol})$ & $120.23 \pm 5.07$ & $0.178 \pm 0.024$ & $-14.50 \pm 0.81$ & 89.76 \\
F4 & $\mathrm{CPC}_{4 \mathrm{k}}, 7.09 \mathrm{mg}(1.426 \mu \mathrm{mol})$ & $121.36 \pm 3.57$ & $0.140 \pm 0.008$ & $-13.80 \pm 0.98$ & 88.31 \\
F5 & $\mathrm{CPC}_{6 \mathrm{k}}, 9.94 \mathrm{mg}(1.426 \mu \mathrm{mol})$ & $135.30 \pm 4.69$ & $0.222 \pm 0.008$ & $-16.23 \pm 1.75$ & 86.68 \\
F6 & mPEG $_{2 \mathrm{k}}-\mathrm{DSPE}, 10 \mathrm{mg}$ & $75.10 \pm 1.25$ & $0.262 \pm 0.007$ & $-13.63 \pm 0.45$ & 87.76 \\
F7 & $\mathrm{CPC}_{2 \mathrm{k}}, 10 \mathrm{mg}$ & $130.80 \pm 4.32$ & $0.525 \pm 0.118$ & $-13.80 \pm 0.98$ & 84.07 \\
F8 & $\mathrm{CPC}_{4 \mathrm{k}}, 10 \mathrm{mg}$ & $116.00 \pm 4.06$ & $0.457 \pm 0.036$ & $-15.23 \pm 0.37$ & 87.41 \\
F9 & $\mathrm{CPC}_{6 \mathrm{k}}, 10 \mathrm{mg}$ & $91.75 \pm 3.283$ & $0.238 \pm 0.013$ & $-15.70 \pm 3.50$ & 90.60
\end{tabular}

$20 \mathrm{mg}$ of lecithin and $100 \mu \mathrm{g}$ of Cou- 6 were used within a total volume of $10 \mathrm{~mL}$ for F1-F4; $100 \mathrm{mg}$ of lecithin and $100 \mu \mathrm{g}$ of Cou-6 were used within a total volume of $10 \mathrm{~mL}$ for $\mathrm{F} 5-\mathrm{F} 8$.

Naked liposomes, which were prepared without CPC, possessed a zeta potential of $-18.17 \mathrm{mV}$ while CPC liposomes, as well as $\mathrm{MPEG}_{2 \mathrm{k}}-\mathrm{DSPE}$, displayed only a slightly lower value. This kind of minor variation in charge density may result from the addition of non-ionic polymers of CPC. However, the variation of PEG moieties in weight or MW did not induce the change in the zeta potential of liposomes.
From a thermodynamics perspective, both cholesterol moieties in a CPC molecule tend to anchor into the lipid bilayer so that the Gibbs energy of the whole system is minimized. If so, PEG chains would present a ring-shaped, specifically speaking, twisted-ring-shaped rather than linear conformation (Fig. 1). A double shell structure, which might be attributed to the lipid membrane and PEG layer, respectively, can be seen in $\mathrm{CPC}_{6 \mathrm{k}}$
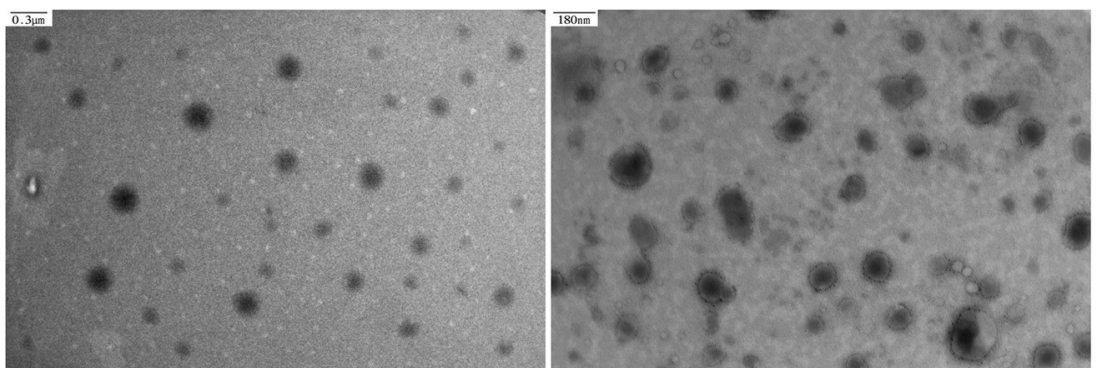

Fig. 6 TEM images of $\mathrm{MPEG}_{2 \mathrm{k}}-\mathrm{DSPE}$ liposomes (left) and $\mathrm{CPC}_{6 \mathrm{k}}$ liposomes (right). 


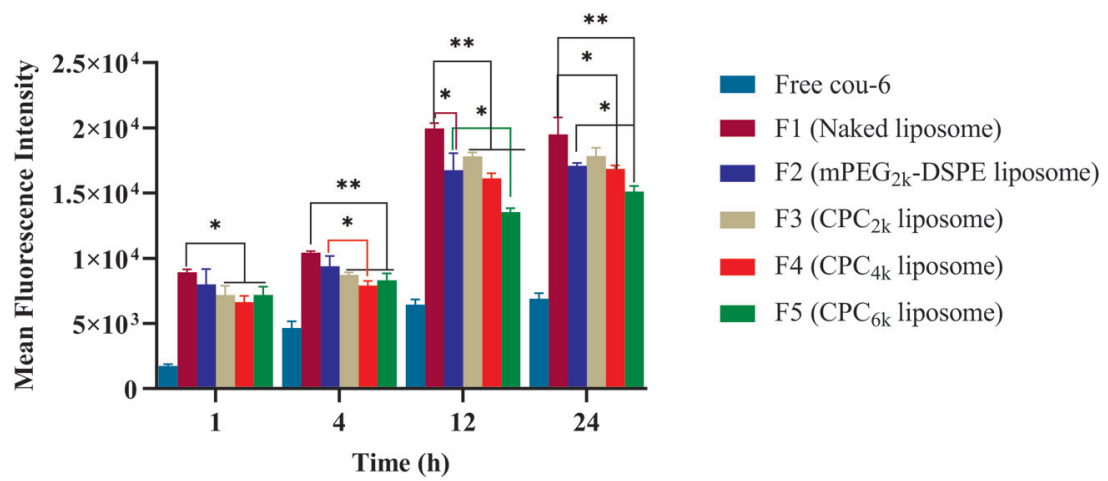

Fig. 7 Mean fluorescence intensity of different kinds of liposomes in RAW264.7 cells. Comparison was made with the $m P E G_{2 k}-D S P E$ group ( ${ }^{*} p<0.05$; $\left.{ }^{* \star} p<0.01 ;{ }^{* \star *} p<0.001\right)$

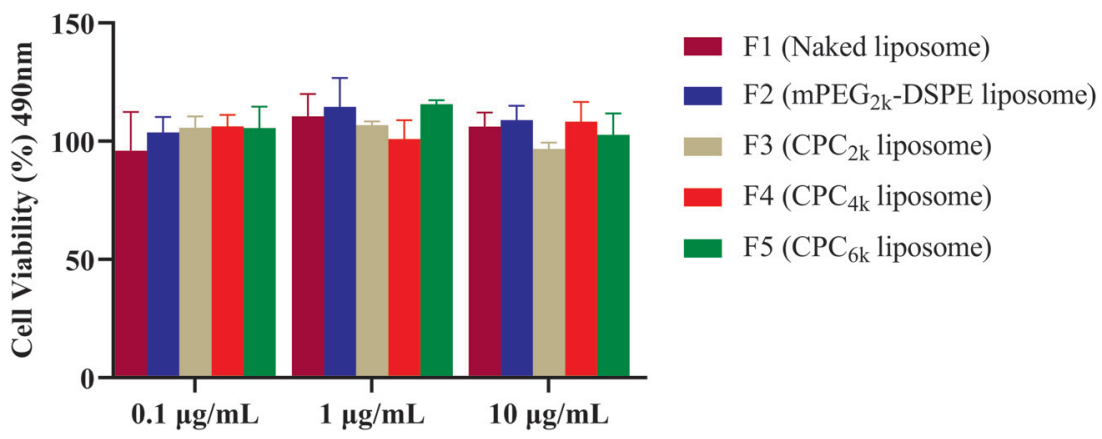

Fig. 8 Cell viability exposed to different kinds of liposomes at various concentrations of CPC.

liposomes but not in other kinds of liposomes (Fig. 6). Under a given resolution of TEM, it is possible that the PEG layer could not be observed unless it is thick enough. Unfortunately, neither the TEM image nor any other widely used spectrum method is adequate to directly differentiate the ring-like conformation from that of linear type.

\subsection{Cellular uptake studies}

All kinds of liposomes showed high Cou-6 loading capacity ranging from $84-90 \%$ (Table 1). As a lipophilic fluorescent probe, Cou- 6 tends to be distributed into the liposomal membrane rather than the water phase. In order to rule out the false positive of cell uptake caused by the leakage of Cou- 6 , the leakage rate was tested before cellular uptake. $\mathrm{CPC}_{4 \mathrm{k}}, \mathrm{mPEG}_{2 \mathrm{k}}-\mathrm{DSPE}$ and naked liposomes showed low enough leakage rates $(0.17 \%, 0.18 \%$ and $0.16 \%$, respectively), so the leakage of Cou- 6 would not obviously affect cellular uptake.

With Cou-6 as the fluorescent probe, different kinds of liposomes (F1-F5 in Table 1) were investigated for cellar uptake and the results are presented in the form of fluorescence intensity of cells in Fig. 7. Compared with the naked liposome (F1), the mPEG2k-DSPE liposome did not show significant difference in cellular uptake until $12 \mathrm{~h}$ passed, while CPC liposomes demonstrated a lower cellular uptake than naked liposomes at $1 \mathrm{~h}(p<0.05)$ and extremely significant differences at $4 \mathrm{~h}$ and $12 \mathrm{~h}(p<0.01)$. The degree to which CPC liposomes evade cellular uptake relied on the MW of the PEG moiety. Under the given molar amount of CPC, the cellular uptake efficiency of CPC liposomes was negatively correlated with the MW of PEG moiety at $12 \mathrm{~h}$, which is consistent with previous reports on mPEG derivatives. ${ }^{1,5,16}$ An increase in size usually enhances the cellular uptake. However, the $\mathrm{CPC}_{6 \mathrm{k}}$ liposome, even with a larger size than that of $\mathrm{mPEG}_{2 \mathrm{k}}-\mathrm{DSPE}$, performed better in evading cellular uptake, which implied its superior surface properties for long circulation.

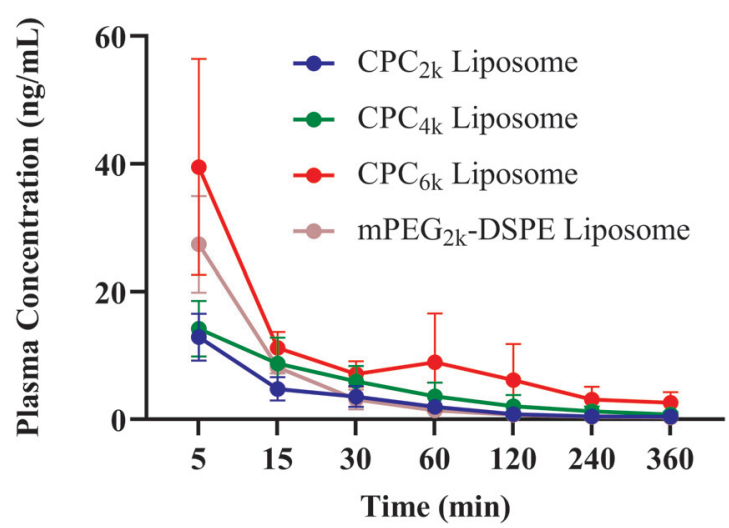

Fig. 9 Blood concentration profiles of Cou-6 after intravenous injection of different liposomes. 
Table 2 Pharmacokinetic parameters of different kinds of liposomes in healthy SD rats

\begin{tabular}{lcccc}
\hline & $\mathrm{mPEG}_{2 \mathrm{k}}-\mathrm{DSPE}$ liposome (F6) & $\mathrm{CPC}_{2 \mathrm{k}}$ liposome (F7) & $\mathrm{CPC}_{4 \mathrm{k}}$ liposome (F8) & $\mathrm{CPC}_{6 \mathrm{k}}$ liposome $(\mathrm{F} 9)$ \\
\hline$T_{1 / 2}(\mathrm{~h})$ & $2.112 \pm 0.8173$ & $1.770 \pm 0.6300$ & $1.728 \pm 0.4969$ & $2.799 \pm 0.3772$ \\
$\mathrm{AUC}\left(\mathrm{ng} \mathrm{L}^{-1} \mathrm{~h}^{-1}\right)$ & $11244 \pm 1860$ & $8657 \pm 2792$ & $15622 \pm 7871$ & $36563 \pm 22284^{*}$ \\
$\mathrm{MRT}(0-t)$ & $0.8992 \pm 0.1006$ & $1.317 \pm 0.2098^{* *}$ & $1.577 \pm 0.2700^{* * *}$ & $1.789 \pm 0.3630^{* *}$ \\
$\mathrm{CL}$ & $3.125 \pm 0.6687$ & $4.267 \pm 1.478$ & $2.562 \pm 1.126$ & $0.9878 \pm 0.4452^{* * *}$ \\
$V$ & $9.006 \pm 1.009$ & $11.31 \pm 6.402$ & $6.232 \pm 3.541$ & $4.050 \pm 1.874^{* *}$ \\
& &
\end{tabular}

\subsection{In vitro cytotoxicity}

Both PEG and cholesterol are nontoxic, so the only possible reason for the toxicity of $\mathrm{CPC}$, if there was, might be its destruction effect on the biomembrane as a surfactant. Cytotoxicity assay was conducted using liposomes prepared with the same formulation as F1 and F6-F9 but without the addition of Cou- 6 . All kinds of liposomes exhibited a relatively high cell viability of above $90 \%$ and no significant difference was observed among any group of liposomes (Fig. 8). So CPCs did not bring significant toxicity at concentrations ranging from $0.1 \mu \mathrm{g} \mathrm{mL} \mathrm{mL}^{-1}$ to $10 \mu \mathrm{g} \mathrm{mL} \mathrm{m}^{-1}$.

\subsection{In vivo PK studies}

The PK studies were performed in SD rats using Cou-6 labeled liposomes (F6-F9, Table 1) and the results are revealed in Fig. 9 and Table 2. In general, the mean residence time (MRT) of all CPC liposomes was significantly higher than that of $\mathrm{MPEG}_{2 \mathrm{k}}{ }^{-}$ DSPE liposomes $(p<0.01)$. The clearance rate $(\mathrm{CL})$ and apparent volume of distribution $(V)$ of CPC liposomes decreased with the increase of MW of PEG chains. So $\mathrm{CPC}_{6 \mathrm{k}}$ showed the lowest value of CL and $V$. Despite a larger size than its $\mathrm{mPEG}_{2 \mathrm{k}}-\mathrm{DSPE}$ counterpart, the $\mathrm{CPC}_{6 \mathrm{k}}$ liposome exhibited a better long-circulating property than any other liposome, which can be seen from the in vivo half-life $\left(T_{1 / 2}\right)$ and AUC.

It is the repetitive units of oxyethylene that endowed PEG chains with hydrophilicity and the resulting stealth capability, which would undoubtedly remain even when the PEG chains were folded into rings. Considering the end group of the PEG chain being the recognition site of MPS uptake, the possible mechanism by which CPC takes effect lies not only in the twisted ring-like conformation but also in the both hidden ends of the PEG chain. CPC could provide a new alternative for stealth liposomes to be drug carriers or imaging agents.

\section{Conclusions}

Derivatives of PEG are gold standards for 'stealth polymers' in liposomes. Different from PEG derivatives in which one end can move freely outside the nanoparticle surface, CPC could anchor both ends into the liposomal membrane to present a twisted-ring-shaped PEG chain, theoretically. In vitro and in vivo experiments in this work presented low toxicity and successful application of CPC liposomes in evading the immune clearance and prolonging the circulation time. Yet, in order to evaluate the potential of CPC as a candidate for stealth polymers, further studies are required to clarify the features of CPC in drug loading and the mechanism of long circulation, especially the interaction between CPC and the immune system.

\section{Conflicts of interest}

There are no conflicts of interest to declare.

\section{Acknowledgements}

This work was supported by the Shandong Provincial Natural Science Foundation, China (No. ZR2018LH023) and the Academic Promotion Program of Shandong First Medical University (Grant No. 2019QL011).

\section{References}

1 S. Y. Fam, C. F. Chee, C. Y. Yong, K. L. Ho, A. R. Mariatulqabtiah and W. S. Tan, Nanomaterials, 2020, 10, 787.

2 S. Deodhar and A. K. Dash, Ther. Delivery, 2018, 9, 857-872.

3 T. Lorson and E. Wegener, et al., Biomaterials, 2018, 178, 204-280.

4 D. Liu, Q. Zhang, J. Wang, S. Guan, D. Cai and J. Liu, Carbohydr. Polym., 2021, 257, 117572.

5 D. Yadav and H. K. Dewangan, J. Biomater. Sci., Polym. Ed., 2021, 32, 266-280.

6 M. Gao, J. Deng, F. Liu, A. Fan, Y. Wang, H. Wu, D. Ding, D. Kong, Z. Wang, D. Peer and Y. Zhao, Biomaterials, 2019, 223, 119486.

7 Y. Yao, P. E. Saw, Y. Nie, P. P. Wong, L. Jiang, X. Ye, J. Chen, T. Ding, L. Xu, H. Yao, H. Hu and X. Xu, J. Mater. Chem. B, 2019, 7, 576-585.

8 J. Hu, Y. Sheng, J. Shi, B. Yu, Z. Yu and G. Liao, Curr. Drug Metab., 2018, 19, 723-738.

9 J. Jiao, X. Luo, C. Wang, X. Jiao, M. Liu, X. Liu, L. Wei, Y. Deng and Y. Song, AAPS PharmSciTech, 2020, 21, 106.

10 S. M. D'Addio, S. Baldassano, L. Shi, L. Cheung, D. H. Adamson, M. Bruzek, J. E. Anthony, D. L. Laskin, P. J. Sinko and R. K. Prud'homme, J. Controlled Release, 2013, 168, 41-49.

11 H. Ren, Y. He, J. Liang, Z. Cheng, M. Zhang, Y. Zhu, C. Hong, J. Qin, X. Xu and J. Wang, ACS Appl. Mater. Interfaces, 2019, 11, 20304-20315.

12 S. Y. Xu, H. Su, X. Y. Zhu, X. Y. Li, J. Li, X. Chen, Q. Wang, R. Y. Hao and X. Y. Yan, J. Liposome Res., 2021, 1-12, DOI: 10.1080/08982104.2021.1884093. 
13 X. Ke, W. Lin, X. Li, H. Wang, X. Xiao and Z. Guo, Drug Delivery, 2017, 24, 1680-1689.

14 Q. Wang, L. He, D. Fan, W. Liang and J. Fang, J. Mater. Chem. B, 2020, 8, 1841-1851.

15 F. Mastrotto, C. Brazzale, F. Bellato, S. De Martin, G. Grange, M. Mahmoudzadeh, A. Magarkar, A. Bunker,
S. Salmaso and P. Caliceti, Mol. Pharmaceutics, 2020, 17, 472-487.

16 M. Liu, Y. Chu, H. Liu, Y. Su, Q. Zhang, J. Jiao, M. Liu, J. Ding, M. Liu, Y. Hu, Y. Dai, R. Zhang, X. Liu, Y. Deng and Y. Song, Mol. Pharmaceutics, 2020, 17, 1059-1070. 